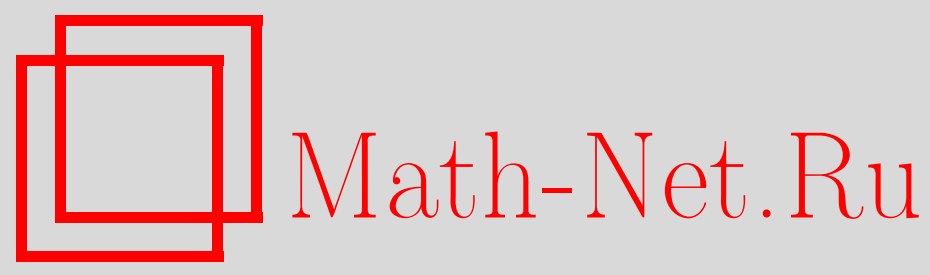

В. В. Басов, Бифуркация инвариантного тора коразмерности единица, Матем. заметки, 2001, том 69, выпуск 1, 3-17

DOI: https://doi.org/10.4213/mzm479

Использование Общероссийского математического портала Math-Net.Ru подразумевает, что вы прочитали и согласны с пользовательским соглашением http://www. mathnet.ru/rus/agreement

Параметры загрузки:

IP: 54.237 .59 .107

26 апреля 2023 г., 15:22:42

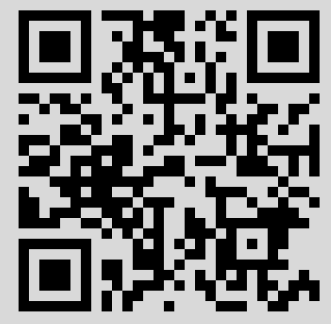




\title{
БИФУРКАЦИЯ ИНВАРИАНТНОГО ТОРА КОРАЗМЕРНОСТИ ЕДИНИЦА
}

\author{
B. B. Басов
}

Предлагается процесс конструктивного построения классов вещественных систем диффференциальных уравнений порядка $2^{d}(d \geqslant 1)$, в том числе полиномиальных, в которых при всех достаточно малых положительных значениях параметра происходит бифуркация рождения инвариантного тора размерности на единицу меньшей, чем размерность системы.

Библиография: 3 названия.

0. Введение. Рассмотрим вещественную автономную систему $2^{d}$ дифференциальных уравнений $(d \geqslant 1)$ с мальм положительным параметром $\varepsilon$

$$
\dot{x}=A x+X(x, \varepsilon),
$$

в которой $x=\left(x_{1}, \ldots, x_{2^{d}}\right) ; A$ - постоянная $\left(2^{d} \times 2^{d}\right)$-матрица, имеющая собственные числа $\pm i \lambda_{1}, \ldots, \pm i \lambda_{2}{ }-1$, которые удовлетворяют конечному числу условий несоизмеримости; вектор-функция $X$ непрерывна и достаточное число раз непрерывно дифференцируема по $x, \varepsilon ; X(\sqrt{\varepsilon} x, \varepsilon)=\varepsilon X^{*}(x, \varepsilon)$. Таким образом, $X(0,0)=0$, и система $(0)$ по теореме о неявной функции имеет при каждом достаточно малом $\varepsilon \geqslant 0$ положение равновесия $x^{*}(\varepsilon)$. Очевидно, что $x^{*}(0)=0$ и $x^{*}(\varepsilon) \rightarrow 0$ при $\varepsilon \rightarrow 0$.

Целью работы является построение класса систем вида $(0)$, имеющих при каждом достаточно малом положительном значении параметра инвариантную поверхность максимальной размерности $2^{d}-1$, гомеоморфную тору. Иными словами, в явном виде будет указан класс систем, в которых при малых $\varepsilon \geqslant 0$ от положения равновесия $x^{*}(\varepsilon)$ ответвляется инвариантньй тор коразмерности единица или, что то же самое, происходит бифуркация рождения инвариантного тора максимальной размерности. При этом константы, в известном смысле задающие амплитуды и частоты колебаний на торе, могут быть выбраны произвольным образом. По ним будут построены младшие члены разложения функции $X(x, \varepsilon)$ из системы $(0)$. Оставшаяся часть функции $X$, начинающаяся с указанного поряда по $x, \varepsilon$ и нужное число раз непрерьвно дифференцируемая по ним, произвольна.

Работа, помимо введения, состоит из трех разделов. В разделе 1 разработана система обозначений и на основе некоего семейства произвольно выбранных констант построены вышеупомянутые младшие члены разложения функции $X(x, \varepsilon)$, которые и предопределяют наличие у системы (0) инвариантной поверхности размерности, на единищу меньшей размерности самой системы. В разделе 2 предложен итерационньй процесс преобразований исходной системы, который после $d$ итераций сводит ее к системе, имеющей 
инвариантную поверхность требуемой размерности. Раздел 3 посвящен доказательству основной теоремы о бифуркации; полученные результаты подробнейшим образом разобраны на примере четырехмерной системы, полиномы из правой части которой непосредственно вычислены, а итерационная замена явно вьписана. И, наконец, приведены комментарии, устанавливающие связь уже имеющихся результатов по данной тематике с результатами, полученными в предлагаемой работе.

\section{1. Обозначения и вспомогательные утверждения.}

1.1. Пусть $\varepsilon$-мальй положительньй параметр; $d$ и $n$-целые числа, $d \geqslant 1,0 \leqslant n \leqslant d$; $x^{(n)}=\left(x_{1}^{(n)}, \ldots, x_{2^{n}}^{(n)}\right)$, в частности, $x^{(0)}=x_{1}^{(0)}$. При $n \geqslant 1$, кроме того, $x^{(n)}=\left(x_{(1)}^{(n)}, x_{(2)}^{(n)}\right)$, где $x_{(1)}^{(n)}=\left(x_{1}^{(n)}, \ldots, x_{2^{n-1}}^{(n)}\right), x_{(2)}^{(n)}=\left(x_{2^{n-1}+1}^{(n)}, \ldots, x_{2^{n}}^{(n)}\right)$. Через $x^{(n)}, y^{(n)}$ будем обозначать вешественные декартовы переменные, а через $r^{(n)}, \varphi^{(n)}$ - полярные координаты, при этом $x^{(n)} \cdot y^{(n)}=\left(x_{1}^{(n)} y_{1}^{(n)}, \ldots, x_{2^{n}}^{(n)} y_{2^{n}}^{(n)}\right), x^{(n)^{2}}=x^{(n)} \cdot x^{(n)}, \widetilde{x}^{(n-1)}=\left(x_{(1)}^{(n)^{2}}+x_{(2)}^{(n)^{2}}\right)$, $\widetilde{\varphi}^{(n)}=\left(\varphi^{(n)}, \ldots, \varphi^{(d-1)}\right)$.

Далее, через $Q^{k}\left(x^{(n)}, \varepsilon\right)$ будем обозначать однородный вещественньй полином степени $k$ от своих переменных. Вовзаимно однозначное соответствие с $Q^{k}$ поставим полином $P^{k}\left(x^{(n)}\right)=Q^{k}\left(x^{(n)}, 1\right)$. Максимальная степень $P^{k}$ может быть, очевидно, меньше $k$. Полином $T^{k}\left(x^{(n)}\right)$ будет всегда начинаться со степени, большей $k$.

Для $n=0, \ldots, d-1$ введем три целочисленные последовательности:

$$
k_{n}=3^{n}, \quad m_{n}=\frac{3^{n}-1}{2}, \quad \theta_{n}=\frac{3^{d}-3^{n+1}}{2} .
$$

Тогда $m_{n+1}=k_{0}+\cdots+k_{n}, \theta_{n}=k_{n+1}+\cdots+k_{d-1}$, т.е.

$$
k_{n}=2 m_{n}+1, \quad m_{n+1}=m_{n}+k_{n}, \quad \theta_{n-1}=\theta_{n}+k_{n} .
$$

Положим $\theta=k_{0}+\cdots+k_{d-1}$. Тогда $\theta_{n}+m_{n+1}=\theta$.

И, наконец, зафиксируем два набора векторов: $c^{(0)}, \ldots, c^{(d-1)}$ с положительными компонентами и $\lambda^{(0)}, \ldots, \lambda^{(d-1)}$ с ненулевыми вещественньми компонентами, - а также ненулевое вещественное число $\lambda$.

1.2. В этом пункте верхний индекс, указьвающий размерность векторов, не изменяется, поэтому он будет отсутствовать.

Положим $z=\left(z_{1}, \ldots, z_{m}\right), p=\left(p_{1}, \ldots, p_{m}\right), z^{p}=z_{1}^{p_{1}} \ldots z_{m}^{p_{m}},|p|=p_{1}+\cdots+p_{m}$, $e^{\nu}=\left(0, \ldots, 1_{\nu}, \ldots, 0\right)$. Множество $m$-мерных векторов с целочисленными неотрицательными компонентами вполне упорядочим соотношением: вектор $p$ предшествует вектору $q$, если положительна первая ненулевая из последовательных разностей $|q|-|p|$, $q_{1}-p_{1}, \ldots, q_{m-1}-p_{m-1}$.

Пусть

$$
P_{j}^{k}(z)=\sum_{p:|p|=0}^{k} \alpha_{j}^{(p)} z^{p}, \quad j=1, \ldots, m .
$$

Число различных степеней полинома степени $k$ от $m$ переменных

$$
\tau_{k, m}=\sum_{\nu=0}^{k} C_{m+\nu-1}^{\nu}
$$


Сделаем замену $z_{j}=\left(c_{j}+x_{j}\right)^{2}$, в которой все компоненты $c_{j}>0$. Тогда

$$
P_{j}^{k}\left((c+x)^{2}\right)=\breve{P}_{j}^{k}(x)+\breve{T}_{j}^{k}(x), \quad j=1, \ldots, m,
$$

где

$$
\breve{P}_{j}^{k}(x)=\sum_{p:|p|=0}^{k} \breve{\alpha}_{j}^{(p)} x^{p},
$$

а степени полиномов $\breve{T}_{j}^{k}(x)$ больше $k$.

Упорядочим семейства $\left\{\alpha_{j}^{(p)}\right\}$ и $\left\{\breve{\alpha}_{j}^{(p)}\right\}$, в результате чего получим векторы $\alpha_{j}$ и $\breve{\alpha}_{j}$ размерности $\tau_{k, m}$. Из равенства (1) вытекает, что $\alpha_{j}$ и $\breve{\alpha}_{j}$ связаны линейным образом. При желании можно вьписать матрицу $A(c)$ размерности $\tau_{k, m} \times \tau_{k, m}$ такую, что $A(c) \alpha_{j}=\breve{\alpha}_{j}$.

УТВЕРЖДЕНИЕ. Имеем $\operatorname{det} A(c) \neq 0$.

ДокАЗАТЕЛЬСтво. Покажем, что нулевому вектору $\breve{\alpha}$ (индекс $j$ опускаем) отвечает единственное решение $\alpha=0$ уравнения $A(c) \alpha=\breve{\alpha}$. Это и будет означать, что $\operatorname{det} A(c) \neq 0$. Доказывать будем индукцией по $|p|=1, \ldots, k$. В последнюю очередь рассмотрим $p=0$.

Продифференцируем (1) по $x_{\nu}$. Тогда

$$
\frac{\partial P^{k}\left((c+x)^{2}\right)}{\partial z_{\nu}} 2\left(c_{\nu}+x_{\nu}\right)=\frac{\partial\left(\breve{P}^{k}(x)+\breve{T}^{k}(x)\right)}{\partial x_{\nu}}, \quad \nu=1, \ldots, m .
$$

Положив $x=0$, получаем

$$
\frac{\partial P^{k}\left(c^{2}\right)}{\partial z_{\nu}} 2 c_{\nu}=\breve{\alpha}^{\left(e^{\nu}\right)} .
$$

Но вектор $\breve{\alpha}$ выбран нулевым, а все компоненты вектора $c$ положительны, поэтому

$$
\frac{\partial P^{k}\left(c^{2}\right)}{\partial z_{\nu}}=0 .
$$

Продифференцируем теперь последнее равенство по $x_{\mu}$. Тогда

$$
\begin{aligned}
& \frac{\partial^{2} P^{k}\left((c+x)^{2}\right)}{\partial z_{\nu} \partial z_{\mu}} 4\left(c_{\nu}+x_{\nu}\right)\left(c_{\mu}+x_{\mu}\right)+\frac{\partial P^{k}\left((c+x)^{2}\right)}{\partial z_{\nu}} 2 \delta_{\nu \mu}=\frac{\partial^{2}\left(\breve{P}^{k}(x)+\breve{T}^{k}(x)\right)}{\partial x_{\nu} \partial x_{\mu}}, \\
& \nu, \mu=1, \ldots, m \text {. }
\end{aligned}
$$

Положим опять $x=0$ и используем тот факт, что все первые производные полинома $P^{k}$ в точке $c^{2}$ равны нулю. Тогда получаем

$$
\frac{\partial^{2} P^{k}\left(c^{2}\right)}{\partial z_{\nu} \partial z_{\mu}} 4 c_{\nu} c_{\mu}=\breve{\alpha}^{\left(e^{\nu}+e^{\mu}\right)},
$$

т.e.

$$
\frac{\partial^{2} P^{k}\left(c^{2}\right)}{\partial z_{i} \partial z_{j}}=0 .
$$


Продолжая этот процесс, нетрудно заметить, что все частные производные $P^{k}$ в точке $c^{2}$ равны нулю.

Переразложив теперь $P^{k}$ по степеням $z-c^{2}$, немедленно убедимся, что $P^{k} \equiv \alpha^{(0)}$, а значит, все $\alpha^{(p)}=0$ при $1 \leqslant|p| \leqslant k$. Но тогда и $\alpha^{(0)}=0$, так как компонента $\breve{\alpha}^{(0)}$ выбрана нулевой. Утверждение доказано.

В результате $\alpha_{j}=A(c)^{-1} \breve{\alpha}_{j}$, т.е., выбрав произвольным образом полином $\breve{P}^{k}(x)$, можно построить $P^{k}(z)$, удовлетворяющий равенству (1).

В дальнейшем вместо (1) потребуется равенство

$$
\left(c_{j}+x_{j}\right) P_{j}^{k}\left((c+x)^{2}\right)=\widetilde{P}_{j}^{k}(x)+T_{j}^{k}(x), \quad j=1, \ldots, m,
$$

которое получается домножением обеих частей $(1)$ на $\left(c_{j}+x_{j}\right)$.

Пусть

$$
\widetilde{P}_{j}^{k}(x)=\sum_{p:|p|=0}^{k} \widetilde{\alpha}_{j}^{(p)} x^{p} .
$$

Тогда $\widetilde{\alpha}_{j}^{(p)}=c_{j} \breve{\alpha}_{j}^{(p)}+\breve{\alpha}_{j}^{\left(p-e^{j}\right)}$, откуда $\breve{\alpha}_{j}^{(p)}=c_{j}^{-1}\left(\widetilde{\alpha}_{j}^{(p)}-\breve{\alpha}_{j}^{\left(p-e^{j}\right)}\right)$. Очевидно, что, задавая произвольно коэффициенты $\widetilde{\alpha}_{j}^{(p)}$, можно последовательно для всех $p$ с $|p|=0, \ldots, k$ получить любые значения коэффициентов $\breve{\alpha}_{j}^{(p)}$. Таким образом, доказана следующая лемма.

ЛЕмма 1. Для всякого полинома $\widetilde{P}_{j}^{k}(x)$ однозначно найдутся полиномы $P_{j}^{k}(z)$ $u T_{j}^{k}(x)$, удовлетворяющие равенству (2). При этом коэффициенты $\widetilde{\alpha}_{j}^{(p)} u \alpha_{j}^{(p)}$ полиномов $\widetilde{P}_{j}^{k}$ и $P_{j}^{k}$ связаны формулами

$$
\alpha_{j}=A(c)^{-1} \breve{\alpha}_{j}, \quad \breve{\alpha}_{j}^{(p)}=c_{j}^{-1}\left(\widetilde{\alpha}_{j}^{(p)}-\breve{\alpha}_{j}^{\left(p-e^{j}\right)}\right)
$$

1.3. Для всякого $n=0, \ldots, d-1, j=1, \ldots, 2^{n}$ и $\nu=0, \ldots, m_{n}$ построим последовательно полиномы $\widetilde{P}_{j}^{k_{n}, \nu}\left(x^{(n)}\right), P_{j}^{k_{n}, \nu}\left(x^{(n)}\right)$ и $Q_{j}^{k_{n}, \nu}\left(\widetilde{x}^{(n)}, \varepsilon\right)$, используя следующие рекуррентные формулы: при $n=0$

$$
\widetilde{P}^{k_{0}, 0}\left(x^{(0)}\right)=\lambda x^{(0)}
$$

при $n \geqslant 1$

$$
\begin{gathered}
\sum_{\nu=0}^{m_{n}} \widetilde{P}_{(1)}^{k_{n}, \nu}\left(x^{(n)}\right) \varepsilon^{\nu}=-\lambda^{(n-1)} \cdot x_{(2)}^{(n)}+x_{(1)}^{(n)} \cdot \sum_{\nu=0}^{m_{n-1}} Q^{k_{n-1}, \nu}\left(\widetilde{x}^{(n-1)}, \varepsilon\right) \varepsilon^{\nu} \\
\sum_{\nu=0}^{m_{n}} \widetilde{P}_{(2)}^{k_{n}, \nu}\left(x^{(n)}\right) \varepsilon^{\nu}=+\lambda^{(n-1)} \cdot x_{(1)}^{(n)}+x_{(2)}^{(n)} \cdot \sum_{\nu=0}^{m_{n-1}} Q^{k_{n-1}, \nu}\left(\widetilde{x}^{(n-1)}, \varepsilon\right) \varepsilon^{\nu} \\
\left(c^{(n)}+x^{(n)}\right) \cdot \sum_{\nu=0}^{m_{n}} P^{k_{n}, \nu}\left(\left(c^{(n)}+x^{(n)}\right)^{2}\right) \varepsilon^{\nu}=\sum_{\nu=0}^{m_{n}}\left(\widetilde{P}^{k_{n}, \nu}\left(x^{(n)}\right)+T^{k_{n}, \nu}\left(x^{(n)}\right)\right) \varepsilon^{\nu} \\
Q^{k_{n}, \nu}\left(\widetilde{x}^{(n)}, \varepsilon\right)=\sum_{p:|p|=0}^{k_{n}} \alpha^{(p), \nu}\left(\widetilde{x}^{(n)}\right)^{p^{k_{n}-|p|}}
\end{gathered}
$$


где $k_{n}, m_{n}, \lambda^{(n)}, c^{(n)}, \widetilde{x}^{(n)}$ определены в п. 1.1, полиномы $T^{k_{n}, \nu}\left(x^{(n)}\right)$ берутся из формулы $(2)$ и начинаются с $k_{n}+1$ порядка, $\alpha^{(p), \nu}-$ коэффициенты полиномов $P^{k_{n}, \nu}\left(\widetilde{x}^{(n)}\right)$, полиномы $Q^{k_{n-1}, \nu}$ имеют размерность $2^{n-1}$.

Предложенные формулы корректны, и полиномы определяются по ним единственным образом. Действительно, максимальная степень полиномов, стоящих в правой части $(3)$, по $x^{(n)}$ равняется $2 k_{n-1}+1$, что не превосходит $k_{n}$, а по $\varepsilon$ равняется $m_{n-1}+k_{n-1}$, что совпадает с $m_{n}$. По существу в правой части (3) происходит переразложение полиномов по степеням $\varepsilon$. Равенство (4) совпадает с равенством (2), поэтому существование и единственность полиномов $P^{k_{n}, \nu}$ вытекает из леммы 1. И, наконец, однородные полиномы $Q^{k_{n}, \nu}$ степени $k_{n}$ в (5) получаются домножением каждого слагаемого $P^{k_{n}, \nu}$ на $\varepsilon$ в соответствующей степени. Таким образом, доказано следующее утверждение.

Лемма 2. Формуль (3)-(5) при $n=0, \ldots, d-1 u \nu=0, \ldots, m_{n}$ однозначно определяют полиномы $Q^{k_{n}, \nu}$ как функиии $\lambda, \lambda^{(0)}, \ldots, \lambda^{(n-1)} u c^{(0)}, \ldots, c^{(n)}$.

\section{2. Теорема о приведении.}

2.1. Зафиксируем натуральное число $d$ и рассмотрим $2^{d}$-мерную вещественную систему с мальп положительным параметром $\varepsilon$

$$
\begin{aligned}
& \dot{x}_{(1)}^{(d)}=-\lambda^{(d-1)} \cdot x_{(2)}^{(d)}+x_{(1)}^{(d)} \cdot S^{(d-1)}\left(\widetilde{x}^{(d-1)}, \varepsilon\right)+\widehat{X}_{(1)}^{(d)}\left(x^{(d)}, \varepsilon\right)+X_{(1)}^{(d)}\left(x^{(d)}, \sqrt{\varepsilon}\right), \\
& \dot{x}_{(2)}^{(d)}=+\lambda^{(d-1)} \cdot x_{(1)}^{(d)}+x_{(2)}^{(d)} \cdot S^{(d-1)}\left(\widetilde{x}^{(d-1)}, \varepsilon\right)+\widehat{X}_{(2)}^{(d)}\left(x^{(d)}, \varepsilon\right)+X_{(2)}^{(d)}\left(x^{(d)}, \sqrt{\varepsilon}\right),
\end{aligned}
$$

где $\widetilde{x}^{(d-1)}=x_{(1)}^{(d)^{2}}+x_{(2)}^{(d)^{2}}$

$$
S^{(d-1)}=\sum_{\nu=0}^{m_{d-1}} Q^{k_{d-1}, \nu}\left(\widetilde{x}^{(d-1)}, \varepsilon\right) \varepsilon^{\nu}
$$

причем $Q^{k_{d-1}, \nu}$ - это однородные вешественные полиномы порядка $k_{d-1}$, построенные в лемме 2 на основе выбранных в п. 1.1 двух наборов векторов $c^{(n)}$ и $\lambda^{(n)}$, двух наборов чисел $k_{n}$ и $m_{n}$ и числа $\lambda \neq 0 ; \widehat{X}^{(d)}$ - произвольный полином, имеющий минимальную степень $k_{d}+1$ и максимальную степень $k_{d}+(d-1)$ по $x^{(d)}$ и $\sqrt{\varepsilon} ; X^{(d)}\left(\sqrt{\varepsilon} x^{(d)}, \sqrt{\varepsilon}\right)=$ $\sqrt{\varepsilon}^{k_{d}+(d-1)+1} X_{*}^{(d)}\left(x^{(d)}, \varepsilon\right)$, функция $X_{*}^{(d)}\left(x^{(d)}, \varepsilon\right)$ непрерьвна при малых значениях аргументов и непрерьвно дифференцируема по $x^{(d)}$. Отметим, что полиномы $Q^{k_{d-1}, \nu}$ имеют суммарную степень $2 k_{d-1}$ по $x^{(d)}$ и $\sqrt{\varepsilon}$, поэтому максимальная степень полиномов $x_{(j)}^{(d)} \cdot S^{(d-1)}(j=1,2)$ равняется $2 k_{d-1}+2 m_{d-1}+1=k_{d}$.

Наложим дополнительно на векторы $\lambda^{(1)}, \ldots, \lambda^{(d-1)}$, когда $d>1$, конечное число условий несоизмеримости. Пусть $p^{(1)}, \ldots, p^{(d-1)}-$ произвольные целочисленные векторы. Тогда для $n=1, \ldots, d-1$ и $k_{n}=3^{n}$

$$
\left(\lambda^{(n)}, p^{(n)}\right) \neq 0 \quad \text { при } 0<\left|p^{(n)}\right| \leqslant k_{n+1}+n .
$$

В этом разделе методом математической индукции будет показано, что система (6) при условии (7) рекуррентной последовательностью замен после $d$ шагов может быть сведена к вещественной $2^{d}$-мерной системе

$$
\begin{aligned}
& \dot{x}^{(0)}=\varepsilon^{\theta}\left(\lambda x^{(0)}+T^{1,0}\left(x^{(0)}\right)+\sqrt{\varepsilon} X_{*}^{(0)}\left(x^{(0)}, \widetilde{\varphi}^{(0)}, \varepsilon\right)\right), \\
& \dot{\varphi}^{(l)}=\varepsilon^{\theta} \lambda^{(l)}+\varepsilon^{\theta+1 / 2} \Phi_{*}^{(l), 0}\left(x^{(0)}, \widetilde{\varphi}^{(0)}, \varepsilon\right), \quad l=0, \ldots, d-1,
\end{aligned}
$$


в которой векторные переменные $\varphi^{(l)}$ имеют размерность $2^{l}$, константы $\theta, \theta_{l}$ определены в п. 1.1, а полином $T^{1,0}$ - в формуле (4) и начинается со второго порядка. Функции, отмеченные звездочкой, определены и непрерывны при малых значениях $x^{(0)}, \varepsilon$ и любых $\widetilde{\varphi}^{(0)}, 2 \pi$-периодичны по $\widetilde{\varphi}^{(0)}$ и непрерьвно дифференцируемы по $x^{(0)}, \widetilde{\varphi}^{(0)}$.

Предваряя доказательство, заметим, что ограничения (7) не использовались при построении полиномов $S^{(d-1)}$. Они будут нужны только для нормализации возникающих на каждом шаге полиномов $\widehat{X}^{(n)}$.

2.2. Предположим, что $d \geqslant 2$ и после $d-1-n$ шагов $(1 \leqslant n \leqslant d-1)$ из системы (6) получена система

$$
\begin{aligned}
\dot{x}_{(1)}^{(n+1)}= & \varepsilon^{\theta_{n}}\left(-\lambda^{(n)} \cdot x_{(2)}^{(n+1)}+x_{(1)}^{(n+1)} \cdot S^{(n)}\left(\widetilde{x}^{(n)}, \varepsilon\right)\right. \\
& \left.+\widehat{X}_{(1)}^{(n+1)}\left(x^{(n+1)}, \varepsilon\right)+X_{(1)}^{(n+1)}\left(x^{(n+1)}, \widetilde{\varphi}^{(n+1)}, \sqrt{\varepsilon}\right)\right), \\
\dot{x}_{(2)}^{(n+1)}= & \varepsilon^{\theta_{n}}\left(+\lambda^{(n)} \cdot x_{(1)}^{(n+1)}+x_{(2)}^{(n+1)} \cdot S^{(n)}\left(\widetilde{x}^{(n)}, \varepsilon\right)\right. \\
& \left.+\widehat{X}_{(2)}^{(n+1)}\left(x^{(n+1)}, \varepsilon\right)+X_{(2)}^{(n+1)}\left(x^{(n+1)}, \widetilde{\varphi}^{(n+1)}, \sqrt{\varepsilon}\right)\right), \\
\dot{\varphi}^{(l)}= & \varepsilon^{\theta_{l}} \lambda^{(l)}+\varepsilon^{\theta+1} \Phi_{*}^{(l), n+1}\left(x^{(n+1)}, \widetilde{\varphi}^{(n+1)}, \sqrt{\varepsilon}\right), \quad l=n+1, \ldots, d-1,
\end{aligned}
$$

в которой

$$
S^{(n)}=\sum_{\nu=0}^{m_{n}} Q^{k_{n}, \nu}\left(\widetilde{x}^{(n)}, \varepsilon\right) \varepsilon^{\nu}, \quad \widehat{X}^{(n+1)}=\sum_{\nu=0}^{\nu_{2}} \varepsilon^{\nu} \sum_{\mu=\mu_{1}}^{\mu_{2}} \widehat{X}^{(n+1), \mu}\left(x^{(n+1)}\right),
$$

где $\nu_{2}=\left[\left(k_{n+1}+n\right) / 2\right], \mu_{1}=\max \left\{0, k_{n+1}+1-2 \nu\right\}, \mu_{2}=k_{n+1}+n-2 \nu$, a $\widehat{X}^{(n+1), \mu}-$ это однородные полиномы от $x^{(n+1)}$ порядка $\mu ; X^{(n+1)}\left(\sqrt{\varepsilon} x^{(n+1)}, \widetilde{\varphi}^{(n+1)}, \sqrt{\varepsilon}\right)$ $=\sqrt{\varepsilon}^{k_{n+1}+n+1} X_{*}^{(n+1)}\left(x^{(n+1)}, \widetilde{\varphi}^{(n+1)}, \varepsilon\right)$, а функции $X_{*}^{(n+1)}$ и $\Phi_{*}^{(l), n+1}$ непрерьвны при малых значениях своих аргументов, $2 \pi$-периодичны по $\widetilde{\varphi}^{(n+1)}$, непрерьвно дифференцируемы по $x^{(n+1)}$ и $\widetilde{\varphi}^{(n+1)}$. Очевидно, что при $n=d-1$, когда не сделано еще ни одного шага, $\varepsilon^{\theta_{n}}=1$, вектор $\widetilde{\varphi}^{(n+1)}$ и уравнения для $\dot{\varphi}^{(l)}$ отсутствуют. Поэтому система (9) совпадает с исходной системой (6).

Опишем $d-n$ шаг. Его цель заключается в преобразовании первых $2^{n+1}$ уравнений системы (9), в результате которого из них будет снова получена система типа (9), но в два раза меньшего порядка. Однако, прежде чем делать необходимую в таких случаях полярную замену, требуется нормализовать специально выделенные для этого в системе (9) полиномы $\widehat{X}^{(n+1)}$, чтобы в полярных координатах зависимость от угловых переменных появлялась только в членах, имеющих достаточно высокий порядок.

ЛЕмма 3. Существует вещественная полиномиальная замена

$$
x^{(n+1)}=y^{(n+1)}+H^{(n+1)}\left(y^{(n+1)}, \varepsilon\right), \quad 1 \leqslant n \leqslant d-1,
$$

минимальная степень полинома $H^{(n+1)}$ которой по $x^{(n+1)} u \sqrt{\varepsilon}$ равняется $k_{n+1}+1$, а максимальная $-k_{n+1}+n$, преобразующая первые $2^{n+1}$ уравнений системы (9) при условиях (7) на вектор $\lambda^{(n)}$ в систему

$$
\begin{aligned}
& \dot{y}_{(1)}^{(n+1)}=\varepsilon^{\theta_{n}}\left(-\lambda^{(n)} \cdot y_{(2)}^{(n+1)}+y_{(1)}^{(n+1)} \cdot\left(S^{(n)}\left(\widetilde{y}^{(n)}, \varepsilon\right)\right.\right. \\
& \left.\left.\quad+\widehat{Y}_{1}^{(n)}\left(\widetilde{y}^{(n)}, \varepsilon\right)\right)-y_{(2)}^{(n+1)} \cdot \widehat{Y}_{2}^{(n)}\left(\widetilde{y}^{(n)}, \varepsilon\right)+Y_{(1)}^{(n+1)}\left(y^{(n+1)}, \widetilde{\varphi}^{(n+1)}, \sqrt{\varepsilon}\right)\right), \\
& \dot{y}_{(2)}^{(n+1)}=\varepsilon^{\theta_{n}}\left(+\lambda^{(n)} \cdot y_{(1)}^{(n+1)}+y_{(2)}^{(n+1)} \cdot\left(S^{(n)}\left(\widetilde{y}^{(n)}, \varepsilon\right)\right.\right. \\
& \left.\left.\quad+\widehat{Y}_{1}^{(n)}\left(\widetilde{y}^{(n)}, \varepsilon\right)\right)+y_{(1)}^{(n+1)} \cdot \widehat{Y}_{2}^{(n)}\left(\widetilde{y}^{(n)}, \varepsilon\right)+Y_{(2)}^{(n+1)}\left(y^{(n+1)}, \widetilde{\varphi}^{(n+1)}, \sqrt{\varepsilon}\right)\right),
\end{aligned}
$$


в которой

$$
\widehat{Y}_{j}^{(n)}=\sum_{\nu=0}^{\nu_{2}} \varepsilon^{\nu} \sum_{\mu=\mu_{1} / 2}^{\left[\mu_{2} / 2\right]} \widehat{Y}_{j}^{(n), \mu}\left(y_{(1)}^{(n+1)^{2}}+y_{(2)}^{(n+1)^{2}}\right), \quad j=1,2
$$

функиия $Y^{(n+1)}$ имеет ту же структуру, что и функиия $X^{(n+1)}$.

ДокАЗАТЕЛЬСТво. Нормализовать систему, имеющую различные благодаря (7) чисто мнимые собственные числа, удобно, когда матрища ее линейной части приведена к диагональному виду, для чего необходимо сделать линейную замену переменных

$$
x_{(1)}^{(n+1)}=\frac{1}{2}\left(u^{(n)}+\bar{u}^{(n)}\right), \quad x_{(2)}^{(n+1)}=\frac{1}{2 i}\left(u^{(n)}-\bar{u}^{(n)}\right),
$$

где $u^{(n+1)}=\left(u^{(n)}, \bar{u}^{(n)}\right)$, а черта - это знак комплексного сопряжения, переводящую уравнения для $\dot{x}^{(n+1)}$ системы $(9)$ в систему

$$
\begin{aligned}
\dot{u}^{(n)}= & \varepsilon^{\theta_{n}}\left(u^{(n)} \cdot\left(i \lambda^{(n)}+S^{(n)}\left(u^{(n)} \cdot \bar{u}^{(n)}, \varepsilon\right)\right)\right. \\
& \left.+\widehat{U}^{(n)}\left(u^{(n+1)}, \varepsilon\right)+U^{(n)}\left(u^{(n+1)}, \breve{\varphi}^{(n+1)}, \sqrt{\varepsilon}\right)\right)
\end{aligned}
$$

в которой остались не выписанными $2^{n}$ уравнений, являющихся комплексно сопряженными к уравнениям (13), а структура функций $\widehat{U}^{(n)}$ и $U^{(n)}$ аналогична структуре функций $\widehat{X}^{(n+1)}$ и $X^{(n+1)}$ системы $(9)$.

Максимальная степень полинома $u^{(n)} \cdot S^{(n)}$ по $u^{(n+1)}$ и $\sqrt{\varepsilon}$ непревосходит $k_{n+1}$, и этот полином уже записан в нормальной форме. Нормализуем полином $\widehat{U}^{(n)}$, которьй также не зависит от $\widetilde{\varphi}^{(n+1)}$. Иными словами, приведем систему (13) к нормальной форме по $u^{(n+1)}$ и $\sqrt{\varepsilon}$ до порядка $k_{n+1}+n$ включительно при помоши полиномиальной замены

$$
u^{(n)}=v^{(n)}+G^{(n)}\left(v^{(n+1)}, \varepsilon\right), \quad \bar{u}^{(n)}=\bar{v}^{(n)}+\bar{G}^{(n)}, \quad 1 \leqslant n \leqslant d-1,
$$

в которой $G^{(n)}$ имеет ту же структуру, что и полином $H^{(n+1)}$ замены $(10)$.

В лемме из работы $[1$, гл. $1, \S 2$, с. 28$]$, в частности, доказьвается, что при условии $(7)$ система (13) заменой (14) может быть приведена к виду

$$
\dot{v}^{(n)}=\varepsilon^{\theta_{n}}\left(v^{(n)} \cdot\left(i \lambda^{(n)}+S^{(n)}\left(v^{(n)} \cdot \bar{v}^{(n)}, \varepsilon\right)+\widehat{V}^{(n)}\left(v^{(n)} \cdot \bar{v}^{(n)}, \varepsilon\right)\right)+V^{(n)}\left(\breve{v}^{(n)}, \widetilde{\varphi}^{(n+1)}, \sqrt{\varepsilon}\right)\right),
$$

где

$$
\widehat{V}^{(n)}=\sum_{\nu=0}^{\nu_{2}} \varepsilon^{\nu} \sum_{\mu=\mu_{1} / 2}^{\left[\mu_{2} / 2\right]} \widehat{V}^{(n), \mu}\left(v^{(n)} \cdot \bar{v}^{(n)}\right)
$$

a $V^{(n)}$ представляет собой сумму функции $U^{(n)}$, в которую подставлена $(14)$, и некоторого полинома, степень которого по $v^{(n+1)}$ и $\sqrt{\varepsilon}$ ограничена снизу числом $k_{n+1}+n+1$. Поэтому $V^{(n)}$ может быть записана в виде

$$
V^{(n)}\left(\sqrt{\varepsilon} v^{(n+1)}, \widetilde{\varphi}^{(n+1)}, \sqrt{\varepsilon}\right)=\sqrt{\varepsilon}^{k_{n+1}+n+1} V_{*}^{(n)}\left(v^{(n+1)}, \widetilde{\varphi}^{(n+1)}, \varepsilon\right) .
$$

Для того чтобы завершить доказательство леммы 3 , остается овеществить систему (15), т.е. сделать линейную замену

$$
v^{(n)}=y_{(1)}^{(n+1)}+i y_{(2)}^{(n+1)}, \quad \bar{v}^{(n)}=y_{(1)}^{(n+1)}-i y_{(2)}^{(n+1)},
$$


обратную к (12). В результате будет получена вешественная система (11), в которой $\widehat{Y}_{1}^{(n)}=\operatorname{Re} \widehat{V}^{(n)}, \widehat{Y}_{2}^{(n)}=\operatorname{Im} \widehat{V}^{(n)}$, а замена (10) представляет собой суперпозицию замен (12), (14) и (16). Лемма 3 доказана.

Надо отметить, что поскольку $\widehat{Y}_{j}^{(n)}$ - полиномы по целым степеням $\varepsilon$ и числа $k_{n+1}+1$ всегда четные, то минимальная степень этих полиномов по $y^{(n+1)}$ и $\sqrt{\varepsilon}$ как раз равняется $k_{n+1}+1$. Например, в системе $(11)$ при $n=1$ полиномы $\widehat{Y}_{j}^{(n)}$ по этой причине отсутствуют.

Сделаем теперь в системе (11) полярную замену переменных

$$
y_{(1)}^{(n+1)}=r^{(n)} \cdot \cos \varphi^{(n)}, \quad y_{(2)}^{(n+1)}=r^{(n)} \cdot \sin \varphi^{(n)} \text {. }
$$

Тогда $r^{(n)^{2}}=\widetilde{y}^{(n)}$ и из $(11)$ получим систему

$$
\begin{aligned}
\dot{r}^{(n)} & =\varepsilon^{\theta_{n}}\left(r^{(n)} \cdot\left(S^{(n)}\left(r^{(n)^{2}}, \varepsilon\right)+\widehat{Y}_{1}^{(n)}\left(r^{(n)^{2}}, \varepsilon\right)\right)+R^{(n)}\left(r^{(n)}, \varphi^{(n)}, \widetilde{\varphi}^{(n+1)}, \sqrt{\varepsilon}\right)\right), \\
r^{(n)} \cdot \dot{\varphi}^{(n)} & =\varepsilon^{\theta_{n}}\left(r^{(n)} \cdot\left(\lambda^{(n)}+\widehat{Y}_{2}^{(n)}\left(r^{(n)^{2}}, \varepsilon\right)\right)+\Phi^{(n)}\left(r^{(n)}, \varphi^{(n)}, \widetilde{\varphi}^{(n+1)}, \sqrt{\varepsilon}\right)\right),
\end{aligned}
$$

в которой

$R^{(n)}=Y_{(1)}^{(n+1)}\left(r^{(n)} \cdot \cos \varphi^{(n)}, r^{(n)} \cdot \sin \varphi^{(n)}, \widetilde{\varphi}^{(n+1)}, \sqrt{\varepsilon}\right) \cdot \cos \varphi^{(n)}+Y_{(2)}^{(n+1)}(\cdots) \cdot \sin \varphi^{(n)}$,

а функция $\Phi^{(n)}$ записьвается аналогично.

Возьмем произвольно выбранньй вектор $c^{(n)}$ с положительными компонентами и сделаем расщепляющую замену переменных

$$
r^{(n)}=\sqrt{\varepsilon}\left(c^{(n)}+x^{(n)}\right),
$$

где $\left|x_{j}^{(n)}\right|<\left|c_{j}^{(n)}\right|$ при $j=1, \ldots, 2^{n}$.

В результате замены (19) из каждого слагаемого однородных полиномов $Q^{k_{n}, \nu}(\varepsilon \times$ $\left.\left(c^{(n)}+x^{(n)}\right)^{2}, \varepsilon\right)$, входящих в функцию $S^{(n)}$ системы $(18)$, стало возможно вынести $\varepsilon^{k_{n}}$, после чего они согласно (5) превратились в полиномы $P^{k_{n}, \nu}\left(\left(c^{(n)}+x^{(n)}\right)^{2}\right)$. Полиномы $\widehat{Y}_{j}^{(n)}$ стали начинаться по $\sqrt{\varepsilon}$ с порядка $k_{n+1}+1$, а функции $R^{(n)}, \Phi^{(n)}-$ с порядка $k_{n+1}+n+1$. Поэтому полученные после замены (19) уравнения для $\dot{x}^{(n)}$ можно сократить на $\sqrt{\varepsilon}$ и из всех слагаемых правой части вьнести $\sqrt{\varepsilon}^{2 k_{n}}$, учитьвая, что $k_{n+1}=2 k_{n}+k_{n}$ и $n \geqslant 1$. В свою очередь, в уравнениях для $\dot{\varphi}^{(n)}$ после деления на $\sqrt{\varepsilon}\left(c^{(n)}+x^{(n)}\right)$ полученная из $\widehat{Y}_{2}^{(n)}+r^{(n)^{-1}} \cdot \Phi^{(n)}$ функция будет иметь минимальньй порядок по $\sqrt{\varepsilon}$, равньй $k_{n+1}+1$. Используем также обозначение из п. 1.1 , соглано которому $\left(\varphi^{(n)}, \widetilde{\varphi}^{(n+1)}\right)=\widetilde{\varphi}^{(n)}$. Тогда полученную из $(18)$ систему можно записать в виде

$$
\begin{aligned}
\dot{x}^{(n)}= & \varepsilon^{\theta_{n}+k_{n}}\left(\left(c^{(n)}+x^{(n)}\right) \cdot \sum_{\nu=0}^{m_{n}} P^{k_{n}, \nu}\left(\left(c^{(n)}+x^{(n)}\right)^{2}\right) \varepsilon^{\nu}\right. \\
& \left.+\widehat{X}_{2}^{(n)}\left(x^{(n)}, \varepsilon\right)+X^{(n)}\left(x^{(n)}, \widetilde{\varphi}^{(n)}, \sqrt{\varepsilon}\right)\right), \\
\dot{\varphi}^{(n)}= & \varepsilon^{\theta_{n}}\left(\lambda^{(n)}+\sqrt{\varepsilon}^{k_{n+1}+1} \Phi_{*}^{(n), n}\left(x^{(n)}, \widetilde{\varphi}^{(n)}, \varepsilon\right)\right),
\end{aligned}
$$


где в полином $\widehat{X}_{2}^{(n)}$ собраны все слагаемые многочлена

$$
\left(c^{(n)}+x^{(n)}\right) \cdot \widehat{Y}_{1}^{(n)}\left(\varepsilon\left(c^{(n)}+x^{(n)}\right)^{2}, \varepsilon\right) \varepsilon^{-k_{n}},
$$

имеющие суммарную степень по $x^{(n)}$ и $\sqrt{\varepsilon}$ не вьше $k_{n}+(n-1)$. Остальные слагаемые этого многочлена вместе с $R^{(n)}$ образуют функцию $X^{(n)}$. Поэтому $\widehat{X}_{2}^{(n)}$ имеет минимальную степень $\sqrt{\varepsilon}$, равную $k_{n}+1$, а

$$
X^{(n)}\left(\sqrt{\varepsilon} x^{(n)}, \widetilde{\varphi}^{(n)}, \sqrt{\varepsilon}\right)=\sqrt{\varepsilon}^{k_{n}+(n-1)+1} X_{*}^{(n)}\left(x^{(n)}, \widetilde{\varphi}^{(n)}, \varepsilon\right) .
$$

Разобьем уравнение для $\dot{x}^{(n)}$ на два, положив $\dot{x}^{(n)}=\left(\dot{x}_{(1)}^{(n)}, \dot{x}_{(2)}^{(n)}\right)$, и поступим так же с вектор-функциями правой части. После этого переразложим полиномы $P_{(j)}^{k_{n}, \nu}(j=1,2)$, входящие в систему $(20)$, по степеням $x^{(n)}$, как это сделано в формуле (4). Получившиеся после этого полиномы

$$
\sum_{\nu=0}^{m_{n}} \widetilde{P}_{(j)}^{k_{n}, \nu}\left(x^{(n)}\right) \varepsilon^{\nu}
$$

перепишем в виде (3). В свою очередь, образовавшийся после переразложения (4) полином

$$
\sum_{\nu=0}^{m_{n}} T^{k_{n}, \nu}\left(x^{(n)}\right) \varepsilon^{\nu}
$$

имеющий минимальньй порядок $k_{n}+1$ по $x^{(n)}$, аналогично предыдущему разделим на две части, одну из которых обозначим $\widehat{X}_{1}^{(n)}\left(x^{(n)}, \varepsilon\right)$. Ее порядок по $x^{(n)}$ и $\sqrt{\varepsilon}$ не превышает $k_{n}+(n-1)$. Оставшиеся слагаемые можно добавить в функцию $X^{(n)}$. И, наконец, согласно введенньгм в п. 1.1 обозначениям имеем $\theta_{n}+k_{n}=\theta_{n-1}, k_{n+1}+1=2 m_{n+1}+2$, $\theta_{n}+m_{n+1}=\theta$.

Возврашаясь к системе (9), заметим, что суперпозиция замен (10), (17) и (19) не меняет структуры $2^{n}$ уравнений для $\dot{\varphi}^{(l)}$. $\Phi$ ункции $\Phi_{*}^{(l), n+1}$ в них после подстановки обозначим через $\Phi_{*}^{(l), n}$.

Итак, из системы (9) после замен (10), (17) и (19) получена система

$$
\begin{aligned}
\dot{x}_{(1)}^{(n)}= & \varepsilon^{\theta_{n-1}}\left(-\lambda^{(n-1)} \cdot x_{(2)}^{(n)}+x_{(1)}^{(n)} \cdot S^{(n-1)}\left(\widetilde{x}^{(n-1)}, \varepsilon\right)\right. \\
& \left.+\widehat{X}_{(1)}^{(n)}\left(x^{(n)}, \varepsilon\right)+X_{(1)}^{(n)}\left(x^{(n)}, \widetilde{\varphi}^{(n)}, \sqrt{\varepsilon}\right)\right), \\
\dot{x}_{(2)}^{(n)}= & \varepsilon^{\theta_{n-1}}\left(+\lambda^{(n-1)} \cdot x_{(1)}^{(n)}+x_{(2)}^{(n)} \cdot S^{(n-1)}\left(\widetilde{x}^{(n-1)}, \varepsilon\right)\right. \\
& \left.+\widehat{X}_{(2)}^{(n)}\left(x^{(n)}, \varepsilon\right)+X_{(2)}^{(n)}\left(x^{(n)}, \widetilde{\varphi}^{(n)}, \sqrt{\varepsilon}\right)\right), \\
\dot{\varphi}^{(l)}= & \varepsilon^{\theta_{l}} \lambda^{(l)}+\varepsilon^{\theta+1} \Phi_{*}^{(l), n}\left(x^{(n)}, \widetilde{\varphi}^{(n)}, \sqrt{\varepsilon}\right), \quad l=n, \ldots, d-1,
\end{aligned}
$$

в которой

$$
S^{(n-1)}=\sum_{\nu=0}^{m_{n-1}} Q^{k_{n-1}, \nu}\left(x_{(1)}^{(n)^{2}}+x_{(2)}^{(n)^{2}}, \varepsilon\right) \varepsilon^{\nu}
$$

$\widehat{X}^{(n)}=\widehat{X}_{1}^{(n)}+\widehat{X}_{2}^{(n)} ; X^{(n)}\left(\sqrt{\varepsilon} x^{(n)}, \widetilde{\varphi}^{(n)}, \sqrt{\varepsilon}\right)=\sqrt{\varepsilon}^{k_{n}+(n-1)+1} X_{*}^{(n)}\left(x^{(n)}, \widetilde{\varphi}^{(n)}, \varepsilon\right), \mathrm{a}$ функции $X_{*}^{(n)}$ и $\Phi_{*}^{(l), n}$ точно так же непрерывны при малых значениях своих аргументов, $2 \pi$-периодичны по $\widetilde{\varphi}^{(n)}$ и непрерьвно дифференцируемы по $x^{(n)}$ и $\widetilde{\varphi}^{(n)}$. 
Шаг с номером $d-n$, где $d \geqslant 2,1 \leqslant n \leqslant d-1$, завершен, так как система (21) является копией (9) при замене в последней $n$ на $n-1$.

2.3. Проделав указанным способом $d-1$ шагов, получим систему $(21) \mathrm{c} n=1$, когда $k_{0}=1, m_{0}=0, \lambda_{1}^{(0)}=\lambda^{(0)}$, т.е. (21) вьглядит так:

$$
\begin{aligned}
& \dot{x}_{1}^{(1)}=\varepsilon^{\theta_{0}}\left(-\lambda^{(0)} x_{2}^{(1)}+x_{1}^{(1)} S^{(0)}\left(\widetilde{x}^{(0)}, \varepsilon\right)+X_{1}^{(1)}\left(x^{(1)}, \widetilde{\varphi}^{(1)}, \sqrt{\varepsilon}\right)\right), \\
& \dot{x}_{2}^{(1)}=\varepsilon^{\theta_{0}}\left(+\lambda^{(0)} x_{1}^{(1)}+x_{2}^{(1)} S^{(0)}\left(\widetilde{x}^{(0)}, \varepsilon\right)+X_{2}^{(1)}\left(x^{(1)}, \widetilde{\varphi}^{(1)}, \sqrt{\varepsilon}\right)\right), \\
& \dot{\varphi}^{(l)}=\varepsilon^{\theta_{l}} \lambda^{(l)}+\varepsilon^{\theta+1} \Phi_{*}^{(l), 1}\left(x^{(1)}, \widetilde{\varphi}^{(1)}, \sqrt{\varepsilon}\right), \quad l=1, \ldots, d-1,
\end{aligned}
$$

где $S^{(0)}=Q^{1,0}\left(x_{1}^{(1)^{2}}+x_{2}^{(1)^{2}}, \varepsilon\right)$, а $\widehat{X}^{(1)} \equiv 0$, так как разрьв между функциями $x_{j}^{(1)} S^{(0)}$ и $X_{j}^{(1)}$ исчез.

До сих пор предполагалось, что $d \geqslant 2$. Это позволяло сделать по крайней мере один шаг процесса рекурентных преобразований. При $d=1$ исходная система (6) сразу совпадает с первыми двумя уравнениями системы (22), остальные уравнения в которой просто отсутствуют.

Сделаем завершающий шаг процесса с номером $d$, после которого линейная часть вновь получаемой системы, а в данном случае уравнения, перестанет быть критической. Для этого рассмотрим систему (9) при $n=0$, очевидно совпадающую с системой (22).

В замене $(10)$ из леммы $3 H^{(1)} \equiv 0$, поскольку нормализовьвать стало нечего. Поэтому $x^{(1)}=y^{(1)}$. Вьполним замены $(17)$ и (19) с $n=0$. Тогда из первых двух уравнений системы (22) получим систему

$$
\begin{aligned}
& \dot{x}^{(0)}=\varepsilon^{\theta_{0}+k_{0}}\left(\left(c^{(0)}+x^{(0)}\right) P^{1,0}\left(\left(c^{(0)}+x^{(0)}\right)^{2}\right)+\sqrt{\varepsilon} X_{*}^{(0)}\left(x^{(0)}, \widetilde{\varphi}^{(0)}, \varepsilon\right)\right), \\
& \dot{\varphi}^{(0)}=\varepsilon^{\theta_{0}}\left(\lambda^{(0)}+\sqrt{\varepsilon}^{k_{1}} \Phi_{*}^{(0), 0}\left(x^{(0)}, \widetilde{\varphi}^{(0)}, \varepsilon\right)\right),
\end{aligned}
$$

являющуюся аналогом системы $(20)$ для $n=0$, но в которой степень $\sqrt{\varepsilon}$ при функциях, отмеченных звездочной, оказалась на единицу меньше ожидаемой. Поскольку $\theta_{0}+1=\theta$ и справедливы равенства (4), (3) с $n=0$, то первое уравнение системы (23) в точности совпадает с первым уравнением финальной системы (8).

Вовтором уравнении системы $(23) \varepsilon^{\theta_{0}} \sqrt{\varepsilon}^{k_{1}}=\varepsilon^{\theta+1 / 2}$. А в уравнениях для $\dot{\varphi}^{(l)}$ системы $(22)$ после подстановки в них новых переменных $x^{(0)}, \varphi^{(0)}$ “лишний” множитель $\sqrt{\varepsilon}$ просто занесем в функции $\Phi_{*}^{(l), 1}$ и стандартно переобозначим их через $\Phi_{*}^{(l), 0}$. В результате получаются оставшиеся $2^{d}-1$ уравнений системы $(8)$.

Приведенные рассуждения позволили доказать следующую теорему.

ТЕОрема 1. Пусть в вещественной системе (6) $d \geqslant 1$ и выполняются условия несоизмеримости (7). Тогда существует рекуррентная последовательность вещественных замен переменных

$$
\begin{aligned}
x^{(n+1)}=( & \left.\sqrt{\varepsilon}\left(c^{(n)}+x^{(n)}\right) \cdot \cos ^{(n)}, \sqrt{\varepsilon}\left(c^{(n)}+x^{(n)}\right) \cdot \sin ^{(n)}\right) \\
& +H^{(n+1)}\left(\left(\sqrt{\varepsilon}\left(c^{(n)}+x^{(n)}\right) \cdot \cos ^{(n)}, \sqrt{\varepsilon}\left(c^{(n)}+x^{(n)}\right) \cdot \sin ^{(n)}\right), \varepsilon\right)
\end{aligned}
$$

$c 0 \leqslant n \leqslant d-1$, являющаяся суперпозицией замен (10), (17) u (19), которая преобразует систему (6) в систему (8). 


\section{3. Теорема о бифуркации, пример, комментарий.}

3.1. Tеорема 2. Пусть $d \geqslant 1 ; k_{n}=3^{n}, m_{n}=\left(3^{n}-1\right) / 2, \theta_{n}=\left(3^{d}-3^{n+1}\right) / 2$ $(0 \leqslant n \leqslant d-1)$. Выберем $c^{(0)}, \ldots, c^{(d-1)}-$ произвольные векторы с положительными компонентами, $\lambda^{(1)}, \ldots, \lambda^{(d-1)}$ - произвольные вещественные векторы $c$ ненулевыми компонентами, удовлетворяюшие конечному числу условий несоизмеримости (7), и вещественные числа $\lambda^{(0)}, \lambda \neq 0$. Тогда в $2^{d}$-мерной системе (6), полином $S^{d-1}$ которой построен в лемме 2 , а функиии $\widehat{X}^{(d)}, X^{(d)}$ произвольнь, при всех достаточно малых положительных значениях параметра є происходит бифуркация рождения инвариантного тора максимальной размерности $2^{d}-1$. Векторы амплитуд и частот колебаний на нем при малых $\varepsilon$ близки к векторам с компонентами $\varepsilon^{(d-n) / 2} c^{(n)} u \varepsilon^{\theta_{n}} \lambda^{(n)}$ соответственно $(n=d-1, \ldots, 0)$.

ДокАЗАТЕльство. В теореме $12^{d}$-мерная система (6) рекуррентной последовательностью замен (24) после $d$ шагов сведена к системе (8). Сделаем масштабирующую замену

$$
x^{(0)}=\sqrt{\varepsilon} z .
$$

Тогда из системе (8) получим систему

$$
\begin{aligned}
\dot{z} & =\varepsilon^{\theta}\left(\lambda z+Z_{*}\left(\varepsilon^{1 / 4} z, \widetilde{\varphi}^{(0)}, \varepsilon\right)\right), \\
\dot{\varphi}^{(l)} & =\varepsilon^{\theta_{l}} \lambda^{(l)}+\varepsilon^{\theta+1 / 4} \Phi_{*}\left(\varepsilon^{1 / 4} z, \widetilde{\varphi}^{(0)}, \varepsilon\right), \quad l=0, \ldots, d-1,
\end{aligned}
$$

где $Z_{*}, \Phi_{*}$ - непрерывные класса $C_{x^{(0)}, \varphi^{(0)}, \ldots, \varphi^{(d-1)}}^{1}$ при малых $z$ и $\varepsilon, 2 \pi$-периодические по компонентам вектора $\widetilde{\varphi}^{(0)}$ функции.

Множитель $\varepsilon^{1 / 4}$ вместо $\varepsilon^{1 / 2}$ появился в системе $(26)$ только из-за подстановки замены (25) в полином $T^{1,0}$ системы (8). При желании этого можно всегда избежать, сделав предварительно в системе (8) аналитическую в нуле замену переменных, которая аннулирует $T^{1,0}$. Однако, в этом нет необходимости, так как система (26) является частным случаем системы (4) из работы $[1$, гл. $1, \S 3$, с. 32$]$, замена $\varepsilon^{1 / 2}$ в которой на $\varepsilon^{1 / 4}$ не существенна. Согласно теореме, доказанной в $[1$, с. 31$]$ и являющейся одной из модификаций леммы Хейла [2], система (26) при всех достаточно малых положительных $\varepsilon$ имеет инвариантное многообразие, задаваемое уравнением $z=F\left(\widetilde{\varphi}^{(0)}, \varepsilon\right)$, где функция $F$ непрерывная и $2 \pi$-периодическая по компонентам вектора $\left(\varphi^{(0)}, \ldots, \varphi^{(d)}\right)$ размерности $2^{d}-1$. Но тогда исходная система (6) обладает инвариантной поверхностью, гомеоморфной тору коразмерности единица, которая получается из инвариантной поверхности $x^{(0)}=\sqrt{\varepsilon} F\left(\widetilde{\varphi}^{(0)}, \varepsilon\right)$ системы $(8)$ при помоши рекуррентных преобразований $(24)$ и, очевидно, стягивается к нулю при $\varepsilon \rightarrow 0$. При этом вектор амплитуд колебаний на торе сколь угодно близок при малых $\varepsilon$ к вектору $\left(\varepsilon^{1 / 2} c^{(d-1)}, \ldots, \varepsilon^{d / 2} c^{(0)}\right)$, так как $H^{(n+1)}$ в $(24)$ и инвариантная поверхность системы (8) имееют положительный порядок по $\varepsilon$. А из вида уравнений для $\dot{\varphi}^{(l)}$ системы (8) вытекает, что вектор частот колебаний при малых $\varepsilon$ близок к вектору $\left(\varepsilon^{\theta_{d-1}} \lambda^{(d-1)}, \ldots, \varepsilon^{\theta_{0}} \lambda^{(0)}\right) /(2 \pi)$. Теорема 2 доказана.

СЛЕДСТВИЕ. При любом достаточно малом положительном в полиномиальная $2^{d}$-мерная система

$$
\begin{aligned}
& \dot{x}_{(1)}^{(d)}=-\lambda^{(d-1)} \cdot x_{(2)}^{(d)}+x_{(1)}^{(d)} \cdot S^{(d-1)}\left(x_{(1)}^{(d)^{2}}+x_{(2)}^{(d)^{2}}, \varepsilon\right) \\
& \dot{x}_{(2)}^{(d)}=+\lambda^{(d-1)} \cdot x_{(1)}^{(d)}+x_{(2)}^{(d)} \cdot S^{(d-1)}\left(x_{(1)}^{(d)^{2}}+x_{(2)}^{(d)^{2}}, \varepsilon\right)
\end{aligned}
$$


имеет инвариантный тор максимальной размерности $2^{d}-1$. Степень полиномов, входящих в правую часть системы $(27)$ по $x^{(d)}$ не превосходит $2 k_{d-1}+1$, по $\varepsilon$ не превосходит $m_{d}$, а их суммарная степень по $x^{(d)}$ и $\varepsilon$ не превосходит $k_{d}-m_{d-1}$.

3.2. В качестве примера построим однородные полиномы $Q^{k_{d-1}, \nu}$ для $d=2$, когда $k_{d-1}=3$, а $\nu=0,1$. Тем самым, будет построено множество четырехмерных систем из теоремы 2 , в которых происходит бифуркация трехмерного тора. Как уже отмечалось в п. 1.2, число различных степеней полинома третьей степени от двух переменных

$$
\tau_{3,2}=\sum_{\nu=0}^{3}(\nu+1)=10
$$

поэтому для нахождения коэффициентов полиномов $Q_{j}^{3, \nu}\left(\widetilde{x}^{(1)}, \varepsilon\right)(j=1,2)$ предстоит решить линейную систему 10-го порядка из леммы 1.

Учитывая, что $d=2$, немного упростим обозначения. Вместо $c^{(0)}, \lambda^{(0)}, x^{(0)}$ будем писать $c_{0}, \lambda_{0}, x_{0}$, а у компонент векторов $c^{(1)}$ и $\lambda^{(1)}$ не будем писать верхний индекс.

Итак, пусть сначала $n=0$. Тогда $m_{n}=0, k_{n}=1$. Из равенств (3) и (4) получаем $\widetilde{P}^{1,0}\left(x_{0}\right)=\lambda x_{0},\left(c_{0}+x_{0}\right) P^{1,0}\left(\left(c_{0}+x_{0}\right)^{2}\right)=\lambda x_{0}+o\left(\left|x_{0}\right|\right)$.

Поскольку $P^{1,0}\left(z_{0}\right)=\alpha^{(0) 0}+\alpha^{(1) 0} z_{0}$, то $\alpha^{(1) 0} c_{0}^{2}+\alpha^{(0) 0}=0,2 c_{0}^{2} \alpha^{(1) 0}=\lambda$, откуда $\alpha^{(1) 0}=\lambda /\left(2 c_{0}^{2}\right), \alpha^{(0) 0}=-\lambda / 2$, и полином $P^{1,0}$ найден. Дополнив его до однородного полинома $Q^{1,0}$ по формуле $(5)$, получаем $S^{(0)}=Q^{1,0}\left(\widetilde{x}_{0}, \varepsilon\right)=\alpha^{(0) 0} \varepsilon+\alpha^{(1) 0} \widetilde{x}_{0}$.

Пусть теперь $n=1$. Тогда в равенствах (3)-(5) векторные полиномы двумерны, $k_{n}=3, m_{n}=1, \nu=0,1, \widetilde{x}_{0}=x_{1}^{(1)^{2}}+x_{2}^{(1)^{2}}$. Из равенства (3) получаем следующие полиномы:

$$
\begin{array}{ll}
\widetilde{P}_{1}^{3,1}\left(x^{(1)}\right)=\alpha^{(0) 0} x_{1}^{(1)}, & \widetilde{P}_{1}^{3,0}\left(x^{(1)}\right)=-\lambda_{0} x_{2}^{(1)}+\alpha^{(1) 0} x_{1}^{(1)}\left(x_{1}^{(1)^{2}}+x_{2}^{(1)^{2}}\right), \\
\widetilde{P}_{2}^{3,1}\left(x^{(1)}\right)=\alpha^{(0) 0} x_{2}^{(1)}, & \widetilde{P}_{2}^{3,0}\left(x^{(1)}\right)=+\lambda_{0} x_{1}^{(1)}+\alpha^{(1) 0} x_{2}^{(1)}\left(x_{1}^{(1)^{2}}+x_{2}^{(1)^{2}}\right) .
\end{array}
$$

Обозначим через $\widetilde{\alpha}_{j}^{\nu}$, где $j=1,2$ и $\nu=0,1$, вектор коэффициентов полинома $\widetilde{P}_{j}^{3, \nu}$, т.е. вектор

$$
\left(\widetilde{\alpha}_{j}^{(0,0) \nu}, \widetilde{\alpha}_{j}^{(1,0) \nu}, \widetilde{\alpha}_{j}^{(0,1) \nu}, \widetilde{\alpha}_{j}^{(2,0) \nu}, \widetilde{\alpha}_{j}^{(1,1) \nu}, \widetilde{\alpha}_{j}^{(0,2) \nu}, \widetilde{\alpha}_{j}^{(3,0) \nu}, \widetilde{\alpha}_{j}^{(2,1) \nu}, \widetilde{\alpha}_{j}^{(1,2) \nu}, \widetilde{\alpha}_{j}^{(0,3) \nu}\right),
$$

и через $\alpha_{j}^{\nu}$ аналогичный вектор коэффициентов полинома $P_{j}^{3, \nu}$. Тогда

$$
\begin{array}{ll}
\widetilde{\alpha}_{1}^{1}=\left(0, \alpha^{(0) 0}, 0,0, \ldots, 0\right), & \widetilde{\alpha}_{1}^{0}=\left(0,0,-\lambda_{0}, 0,0,0, \alpha^{(1) 0}, 0, \alpha^{(1) 0}, 0\right), \\
\widetilde{\alpha}_{2}^{1}=\left(0,0, \alpha^{(0) 0}, 0, \ldots, 0\right), & \widetilde{\alpha}_{2}^{0}=\left(0,+\lambda_{0}, 0,0,0,0,0, \alpha^{(1) 0}, 0, \alpha^{(1) 0}\right) .
\end{array}
$$

Из равенства (4) вытекает, что

$$
A_{j}\left(c_{1}, c_{2}\right) \alpha_{j}^{\nu}=\widetilde{\alpha}_{j}^{\nu}, \quad j=1,2, \quad \nu=0,1
$$


где, например, матрица $A_{1}\left(c_{1}, c_{2}\right)$ равняется

$$
\left(\begin{array}{cccccccccc}
1 & c_{1}^{2} & c_{2}^{2} & c_{1}^{4} & c_{1}^{2} c_{2}^{2} & c_{2}^{4} & c_{1}^{6} & c_{1}^{4} c_{2}^{2} & c_{1}^{2} c_{2}^{4} & c_{2}^{6} \\
0 & 2 c_{1}^{2} & 0 & 4 c_{1}^{4} & 0 & 0 & 6 c_{1}^{6} & 0 & 0 & 0 \\
0 & 0 & 2 c_{1} c_{2} & 0 & 0 & 4 c_{1} c_{2}^{3} & 0 & 0 & 0 & 6 c_{1} c_{2}^{5} \\
0 & 3 c_{1} & 0 & 10 c_{1}^{3} & 0 & 0 & 21 c_{1}^{5} & 0 & 0 & 0 \\
0 & 0 & 2 c_{2} & 0 & 4 c_{1}^{2} c_{2} & 4 c_{2}^{3} & 0 & 8 c_{1}^{4} c_{2} & 8 c_{1}^{2} c_{2}^{3} & 6 c_{2}^{5} \\
0 & 0 & c_{1} & 0 & 0 & 6 c_{1} c_{2}^{2} & 0 & 0 & 0 & 15 c_{1} c_{2}^{4} \\
0 & 1 & 0 & 10 c_{1}^{2} & 0 & 0 & 35 c_{1}^{4} & 0 & 0 & 0 \\
0 & 0 & 0 & 0 & 6 c_{1} c_{2} & 0 & 0 & 20 c_{1}^{3} c_{2} & 12 c_{1} c_{2}^{3} & 0 \\
0 & 0 & 1 & 0 & 2 c_{1}^{2} & 6 c_{2}^{2} & 0 & 4 c_{1}^{4} & 12 c_{1}^{2} c_{2}^{2} & 15 c_{2}^{4} \\
0 & 0 & 0 & 0 & 0 & 4 c_{1} c_{2} & 0 & 0 & 0 & 20 c_{1} c_{2}^{3}
\end{array}\right)
$$

Решив линейные системы (28) при $j=1$ и подставив в решения уже найденные коэффициенты $\alpha^{(\nu) 0}$, получаем вектор

$$
\alpha_{1}^{1}=\left(\frac{19 \lambda}{32},-\frac{35 \lambda}{32 c_{1}^{2}}, 0, \frac{21 \lambda}{32 c_{1}^{4}}, 0,0,-\frac{5 \lambda}{32 c_{1}^{6}}, 0,0,0\right)
$$

и вектор $\alpha_{1}^{0}$ с компонентами

$$
\begin{gathered}
\alpha_{1}^{(0,0) 0}=\frac{3 c_{2}}{16 c_{1}} \lambda_{0}-\frac{c_{1}^{2}-c_{2}^{2}}{16 c_{0}^{2}} \lambda, \quad \alpha_{1}^{(1,0) 0}=\frac{3 \lambda}{16 c_{0}^{2}}, \quad \alpha_{1}^{(0,1) 0}=-\frac{15 \lambda_{0}}{16 c_{1} c_{2}} \\
\alpha_{1}^{(2,0) 0}=-\frac{3 \lambda}{16 c_{0}^{2} c_{1}^{2}}, \quad \alpha_{1}^{(1,1) 0}=\frac{3 \lambda_{0}}{4 c_{1}^{3} c_{2}}-\frac{\lambda}{8 c_{0}^{2} c_{1}^{2}}, \quad \alpha_{1}^{(0,2) 0}=\frac{5 \lambda_{0}}{16 c_{1} c_{2}^{3}}, \\
\alpha_{1}^{(3,0) 0}=\frac{\lambda}{16 c_{0}^{2} c_{1}^{4}}, \quad \alpha_{1}^{(2,1) 0}=-\frac{3 \lambda_{0}}{16 c_{1}^{5} c_{2}}, \\
\alpha_{1}^{(1,2) 0}=-\frac{\lambda_{0}}{16 c_{1}^{3} c_{2}^{3}}+\frac{\lambda}{16 c_{0}^{2} c_{1}^{2} c_{2}^{2}}, \quad \alpha_{1}^{(0,3) 0}=\frac{\lambda_{0}}{16 c_{1} c_{2}^{5}} .
\end{gathered}
$$

Таким образом, полиномы $P_{1}^{3,0}$ и $P_{1}^{3,1}$ построены. Полиномы $P_{2}^{k_{1}, \nu}$ строятся точно так же. При этом оказывается, что полином

$$
P_{1}^{3,1}\left(x^{(1)}\right)=\frac{19 \lambda}{32}-\frac{35 \lambda}{32 c_{1}^{2}} x_{1}^{(1)}+\frac{21 \lambda}{32 c_{1}^{4}} x_{1}^{(1)^{2}}-\frac{5 \lambda}{32 c_{1}^{6}} x_{1}^{(1)^{3}}
$$

не зависит от второй компоненты двумерного вектора $x^{(1)}$.

Теперь остается дополнить полиномы $P_{j}^{3, \nu}$ по формуле (5) до однородных многочленов третьего порядка $Q_{j}^{3, \nu}$. Так, например,

$$
Q_{1}^{3,1}\left(\widetilde{x}^{(1)}, \varepsilon\right)=\frac{19 \lambda}{32} \varepsilon^{3}-\frac{35 \lambda}{32 c_{1}^{2}} \widetilde{x}_{1}^{(1)} \varepsilon^{2}+\frac{21 \lambda}{32 c_{1}^{4}}\left(\widetilde{x}_{1}^{(1)}\right)^{2} \varepsilon-\frac{5 \lambda}{32 c_{1}^{6}}\left(\widetilde{x}_{1}^{(1)}\right)^{3} .
$$

Согласно введенньм в п. 1.1 обозначениям $\widetilde{x}^{(1)}=x_{(1)}^{(2)^{2}}+x_{(2)}^{(2)^{2}}$ или $\widetilde{x}^{(1)}=\left(\widetilde{x}_{1}^{(1)}, \widetilde{x}_{2}^{(1)}\right)=$ $\left(x_{1}^{(2)^{2}}+x_{3}^{(2)^{2}}, x_{2}^{(2)^{2}}+x_{4}^{(2)^{2}}\right)$. Опуская для краткости верхний индекс у компонент вектора $x^{(2)}$, вьпишем в обшем виде все однородные многочлены $Q^{3, \nu}$ :

$$
\begin{aligned}
Q_{j}^{3, \nu}\left(\widetilde{x}^{(1)}, \varepsilon\right)= & \alpha_{j}^{(0,0) \nu} \varepsilon^{3}+\left(\alpha_{j}^{(1,0) \nu}\left(x_{1}^{2}+x_{3}^{2}\right)+\alpha_{j}^{(0,1) \nu}\left(x_{2}^{2}+x_{4}^{2}\right)\right) \varepsilon^{2} \\
& +\left(\alpha_{j}^{(2,0) \nu}\left(x_{1}^{2}+x_{3}^{2}\right)^{2}+\alpha_{j}^{(1,1) \nu}\left(x_{1}^{2}+x_{3}^{2}\right)\left(x_{2}^{2}+x_{4}^{2}\right)\right. \\
& \left.+\alpha_{j}^{(0,2) \nu}\left(x_{2}^{2}+x_{4}^{2}\right)^{2}\right) \varepsilon+\alpha_{j}^{(3,0) \nu}\left(x_{1}^{2}+x_{1}^{2}\right)^{3}+\alpha_{j}^{(2,1) \nu}\left(x_{1}^{2}+x_{1}^{2}\right)^{2}\left(x_{2}^{2}+x_{4}^{2}\right) \\
& +\alpha_{j}^{(1,2) \nu}\left(x_{1}^{2}+x_{1}^{2}\right)\left(x_{2}^{2}+x_{4}^{2}\right)^{2}+\alpha_{j}^{(0,3) \nu}\left(x_{2}^{2}+x_{4}^{2}\right)^{3}
\end{aligned}
$$


где $j=1,2, \nu=0,1$; коэффицшиенты $\alpha_{j}^{\nu}$ подсчитаны вьше и зависят от параметров $c_{0}, c_{1}, c_{2}, \lambda, \lambda_{0}$.

Рассмотрим четырехмерную вешественную систему

$$
\begin{aligned}
& \dot{x}_{1}=-\lambda_{1} x_{3}+x_{1}\left(Q_{1}^{3,0}\left(\widetilde{x}^{(1)}, \varepsilon\right)+\varepsilon Q_{1}^{3,1}\left(\widetilde{x}^{(1)}, \varepsilon\right)\right)+\widehat{X}_{1}\left(x^{(2)}, \varepsilon\right)+X_{1}\left(x^{(2)}, \sqrt{\varepsilon}\right), \\
& \dot{x}_{2}=-\lambda_{2} x_{4}+x_{2}\left(Q_{2}^{3,0}\left(\widetilde{x}^{(1)}, \varepsilon\right)+\varepsilon Q_{2}^{3,1}\left(\widetilde{x}^{(1)}, \varepsilon\right)\right)+\widehat{X}_{2}\left(x^{(2)}, \varepsilon\right)+X_{2}\left(x^{(2)}, \sqrt{\varepsilon}\right), \\
& \dot{x}_{3}=+\lambda_{1} x_{1}+x_{3}\left(Q_{1}^{3,0}\left(\widetilde{x}^{(1)}, \varepsilon\right)+\varepsilon Q_{1}^{3,1}\left(\widetilde{x}^{(1)}, \varepsilon\right)\right)+\widehat{X}_{3}\left(x^{(2)}, \varepsilon\right)+X_{3}\left(x^{(2)}, \sqrt{\varepsilon}\right), \\
& \dot{x}_{4}=+\lambda_{2} x_{2}+x_{4}\left(Q_{2}^{3,0}\left(\widetilde{x}^{(1)}, \varepsilon\right)+\varepsilon Q_{2}^{3,1}\left(\widetilde{x}^{(1)}, \varepsilon\right)\right)+\widehat{X}_{4}\left(x^{(2)}, \varepsilon\right)+X_{4}\left(x^{(2)}, \sqrt{\varepsilon}\right),
\end{aligned}
$$

в которой однородные многочлены $Q_{j}^{3, \nu}$ только что построены; $\widehat{X}^{(d)}-$ произвольньй однородньй полином десятого порядка относительно $x^{(2)}$ и $\sqrt{\varepsilon}$;

$$
X^{(2)}\left(\sqrt{\varepsilon} x^{(2)}, \sqrt{\varepsilon}\right)=\sqrt{\varepsilon}^{11} X_{*}^{(2)}\left(x^{(2)}, \varepsilon\right),
$$

a $X_{*}^{(2)}\left(x^{(2)}, \varepsilon\right)$ - произвольная функция, указанного в теореме 1 класса гладкости.

Суперпозиция замен $(24)$ с $n=1$ и $n=0\left(H^{(1)} \equiv 0\right)$ преобразует систему (29) согласно теореме 1 в систему (8) с $d=2$, которая имеет по теореме 2 трехмерное инвариантное многообразие $x_{0}=\sqrt{\varepsilon} F\left(\varphi_{0}, \varphi_{1}, \varphi_{2}, \varepsilon\right)$. В результате можно в явном виде выписать инвариантное многообразие системы (29), гомеоморфное трехмерному тору:

$$
\begin{aligned}
& x_{1}=\sqrt{\varepsilon}\left(c_{1}+\sqrt{\varepsilon}\left(c_{0}+\sqrt{\varepsilon} F\left(\varphi_{0}, \varphi_{1}, \varphi_{2}, \varepsilon\right)\right) \cos \varphi_{0}\right) \cos \varphi_{1}+H_{1}\left(x^{(2)}, \varepsilon\right), \\
& x_{2}=\sqrt{\varepsilon}\left(c_{2}+\sqrt{\varepsilon}\left(c_{0}+\sqrt{\varepsilon} F\left(\varphi_{0}, \varphi_{1}, \varphi_{2}, \varepsilon\right)\right) \sin \varphi_{0}\right) \cos \varphi_{2}+H_{2}\left(x^{(2)}, \varepsilon\right), \\
& x_{3}=\sqrt{\varepsilon}\left(c_{1}+\sqrt{\varepsilon}\left(c_{0}+\sqrt{\varepsilon} F\left(\varphi_{0}, \varphi_{1}, \varphi_{2}, \varepsilon\right)\right) \cos \varphi_{0}\right) \sin \varphi_{1}+H_{3}\left(x^{(2)}, \varepsilon\right), \\
& x_{4}=\sqrt{\varepsilon}\left(c_{2}+\sqrt{\varepsilon}\left(c_{0}+\sqrt{\varepsilon} F\left(\varphi_{0}, \varphi_{1}, \varphi_{2}, \varepsilon\right)\right) \sin \varphi_{0}\right) \sin \varphi_{2}+H_{4}\left(x^{(2)}, \varepsilon\right),
\end{aligned}
$$

где

$$
\begin{aligned}
H^{(2)}= & H^{(2)}\left(\sqrt{\varepsilon}\left(c^{(1)}+\left(\sqrt{\varepsilon}\left(c_{0}+x_{0}\right) \cos \varphi_{0}, \sqrt{\varepsilon}\left(c_{0}+x_{0}\right) \sin \varphi_{0}\right)\right) \cdot \cos \varphi^{(1)},\right. \\
& \left.\sqrt{\varepsilon}\left(c^{(1)}+x^{(1)}\right) \cdot \sin \varphi^{(1)}, \varepsilon\right) .
\end{aligned}
$$

Разложение полинома $H^{(2)}$ по степеням $\sqrt{\varepsilon}$, как уже было установлено, начинается с одиннадцатого порядка и не может оказьвать существенного влияния на координаты инвариантного тора. Поэтому справедлива следующая теорема.

Теорема 3. В системе (29) при всех достаточно малых полохительных значениях параметра є происходит бифуркаиия трехмерного инвариантного тора (30) с векторами амплитуд и частот колебаний на нем, стремящимися при $\varepsilon \rightarrow 0$ соответственно $\kappa$ векторам $\left(\sqrt{\varepsilon} c_{1}, \sqrt{\varepsilon} c_{2}, \varepsilon c_{0}\right) u\left(\lambda_{1}, \lambda_{2}, \varepsilon^{3} \lambda_{0}\right) /(2 \pi)$, причем константы $c^{(1)}, c^{(0)}$ и $\lambda^{(1)}, \lambda^{(0)}$ выббраны произвольным образом.

ЗАмЕчаниЕ 1. Если в системе $(29)$ выбрать функции $\widehat{X}^{(2)}$ и $X^{(2)}$ тождественно равными нулю, то (29) превратится в четырехмерную полиномиальную систему, порядок которой по $x^{(2)}$ не превосходит 7 , по $\varepsilon-4$, а суммарньй порядок не превосходит 8 . Согласно теореме 3 в системе (29) при каждом малом положительном значении параметра от положения равновесия ответвляется трехмерный инвариантньй тор с наперед заданными амплитудами и частотами колебаний на нем. 
ЗАмЕЧАнИЕ 2. Построенные в примере полиномы $Q^{3, \nu}$ можно использовать для построения систем более высокого порядка и в первую очередь восьмимерных, в которых бифурцирует семимерный тор. Однако, чисто технически сделать это затруднительно, так как уже при $d=3$, когда $k_{2}=9$, а переменных у полиномов $Q^{9, \nu}$ четыре, пришлось бы решать линейную систему из леммы 1 порядка

$$
\tau_{9,4}=\sum_{\nu=0}^{9} \frac{(\nu+1)(\nu+2)(\nu+3)}{6}=715 .
$$

3.3. Обсудим полученные результаты. В основу статьи легла работа Ю. Н. Бибикова [1], в которой, в частности, рассматривались системы вида (0) из введения. Для таких систем был предложен некий рекуррентньй процесс, после каждого шага которого при выполнении ряда условий получалась система, аналогичная исходной, порядок которой уменьшался в два раза или более, а также дифференциальные уравнения для угловых и некритических переменных. Но процесс Бибикова оказался не приспособленным для нахождения инвариантных торов именно максимальной размерности. В ряде случаев он должен автоматически прерваться после первого же шага. Дело в том, что в предложенном Бибиковым процессе все константы $k_{n}$ обязаны равняться единице, т.е. после каждой итерации степень $\varepsilon$ в правой части уравнения для $\dot{x}^{(n)}$ увеличивается только на единицу. В этом случае свободных параметров, требующихся для вьполнения условий, необходимых для продолжения процесса, после первого шага или познее оказывается недостаточно. Поэтому для практического нахождения систем, в которых происходит бифуркация тора максимальной размерности, процесс Бибикова пришлось существенно модифицировать. В частности, полиномы $Q^{k_{d-1}, \nu}$ в лемме 2 построены таким образом, что после каждой итерации они переходят в себя, а члены более высокого порядка не могут их разрушить; уравнения для $\dot{x}^{(n)}$ системы $(21)$ имеют размерность ровно в два раза меньшую, чем предшествуюшие им уравнения для $\dot{x}^{(n+1)}$ системы $(9) ;$ полученная на последнем шаге система (8) удовлетворяет теореме о существовании инвариантного многообразия. Таким образом, множество систем (6) реализует модифицированный процесс на практике.

В заключение отметим, что при $d=1$ имеет место вариант хорошо известного случая бифуркации Хопфа, а при $d=2$ существование четырехмерных систем, в которых бифурцирует трехмерный тор, было доказано в работе [3].

\section{СПИСОК ЦИТИРОВАННОЙ ЛИТЕРАТУРЫ}

[1] Бибиков Ю.Н. Многочастотные нелинейные колебания и их бифуркации. Л.: Ленинградский ун-т, 1991.

[2] Hale J. K. Integral manifolds of perturbed differential systems // Ann. of Math. 1961. V. 73. №3. P. 496-531.

[3] Maffei C., Negrini P., Scalla M. Bifurcation from 2-dimensional to 3-dimensional invariant tori // Ann. di Matematica pura ed applicata. IV. 1989. V. CLV. P. 117-136.

Научно-исследовательский институт математики и механики им. В.И. Смирнова Поступило Санкт-Петербургского государственного университета E-mail : basov@vb2674.spb.edu 